\title{
A Virtual Environment for Conceptual Design in Architecture
}

\author{
Lee Anderson, ${ }^{1}$ James Esser $^{2 \dagger}$ and Victoria Interrante ${ }^{2}$ \\ ${ }^{1}$ Department of Architecture, University of Minnesota, USA \\ ${ }^{2}$ Department of Computer Science, University of Minnesota, USA
}

\begin{abstract}
We present a virtual environment application that has been developed for conceptual design in architecture and seeks to emulate aspects of a typical designer's work area. The environment provides a means of creating and manipulating basic geometry using a kiosk toolbox. More importantly, the environment provides simple means for using imagery and videos developed outside of the environment for use within the environment for both information and design. A DesignStation is provided within the environment to create a work area for the designer, concentrating imagery and information associated with the design as well as an area for reflecting upon, presenting and critiquing the design process. A unique aspect of the environment is the ability to work in more than one scale simultaneously.
\end{abstract}

Categories and Subject Descriptors (according to ACM CCS): I.3.6 [Computer Graphics]: Interactive Techniques Keywords: Virtual Environments, Architectural Design, Conceptual Design.

\section{Introduction}

Computer Aided Design has been of great benefit in all areas of design, but has had limited acceptance in the area of conceptual design in architecture. There are several reasons for this reservation, among them: the limited viewing angle and resolution of a typical workstation screen tends to objectify the space being designed, leading to a tendency to concentrate on external form rather than inhabitable space; the design of the space can be negatively influenced by the shape of the screen and by its strong horizontal and vertical edges; CAD applications tend to be optimized for the creation and manipulation of geometry rather than spatial creation and the manipulation of imagery; and, at a time when the designer is performing a highly integrative act creating a physical presence, the objects being created are sensed only in a limited visual manner and from a static viewing position.

Many of these problems can potentially be overcome with an immersive virtual design environment. Care must be taken, however, so that the designer isn't just placed "on the other side of the screen" in an environment that, though it may be suitable for creating geometry, doesn't otherwise support the design process.

\rceil Now at Cryptic Studios, San Jose
This paper describes an implementation of a virtual environment for conceptual architectural design that, in addition to providing an interactive environment for creating and manipulating geometry, attempts to adapt and enhance elements from traditional design environments that are rich in imagery and information. The objective is to create a place ("DesignStation") within the virtual design environment that the designer can productively "inhabit" and refer to while manipulating both imagery and geometry during a design process. The intention is to support a process of design that includes both creation and evaluation, or "critiquing", and to ensure that this environment interfaces in an easy and uncomplicated way with the world "outside" so that results from aspects of design that may be best handled outside can flow easily into and out of the virtual environment. An additional goal of this environment is to support the design process at any scale and at multiple scales simultaneously.

Research on the virtual environment for conceptual design project is being conducted through the auspices of the Digital Design Consortium at the University of Minnesota. The Digital Design Consortium was founded to explore the application of digital tools to design and involves faculty from the Departments of Architecture and Computer Science. 


\section{Previous Work}

Architectural design has long been a driving application for immersive virtual environments, and many immersive modeling systems have been developed and tested $8,11,18,19,14,12,13,15,20,4$. Similarly, the problem of determining how to best harness the potential of digital tools to facilitate the earliest stages of the conceptual design process is an important topic that has received considerable attention over the years from researchers in both Architecture and Computer Science 9, 5, 7, 6, 16, 17, 10, 21, 23, 22 .

Considerable advances have been made in screen-based CAD and architectural design programs, such as AutoCAD, Architectural Studio, UpFront ${ }^{1}$, SketchUp ${ }^{24}$, and other commercial products. However commercial software developers have as of yet paid little attention to the prospect of immersive virtual environments for design.

\section{Background: Physical Environment}

Our virtual environment application runs on a Dell 650 workstation and is written in Visual C++ with OpenGL. We have examined a number of alternatives for a head-mounted display and are currently expecting to purchase a SEOS HMD 120/40. The environment is implemented using a HiBall 3000 optical tracker with a 24' by 24' trackable area, which is large enough so that there can be significant physical movement within the space being created.

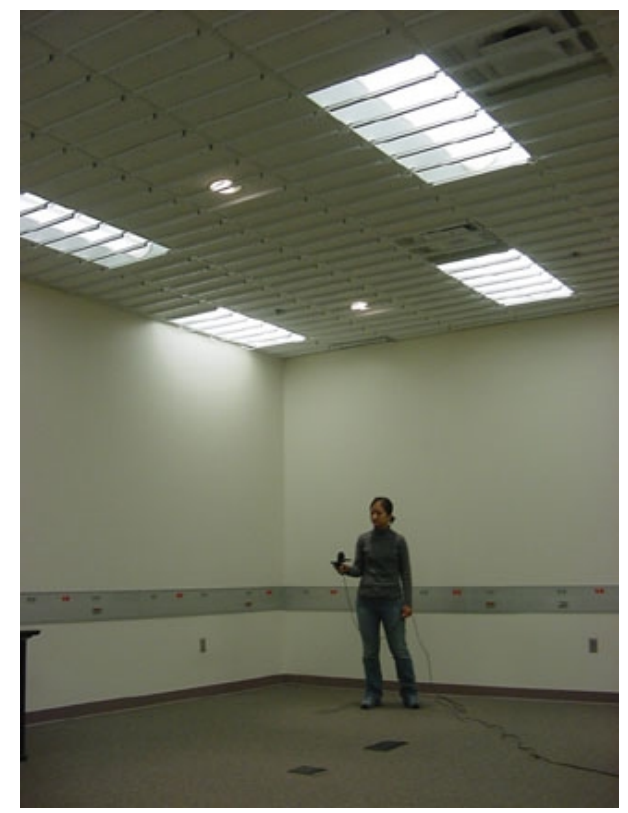

Figure 1: An image of our lab, showing a portion of the tracked area.

In addition to working with an HMD, our system also runs in single screen mono, single screen stereo and dualprojector stereo environments, where we use standard gaming key and mouse conventions for controlling movement and viewing. This allows designers the option to set up elements of their design environment using traditional equipment as an alternative to working exclusively in the virtual environment. In planned studies, the screen environments will be used to compare design process methods of working between the traditional desktop and immersive virtual environment conditions.

\section{The Geometry Tools and Toolbox}

Since we intend to test and continue development of the environment in actual use with a large number of designers posessing different skill levels, a primary goal has been to provide tools that are very quick to learn, easy to use, and forgiving of mistakes.

The current environment supports simple creation, manipulation, copying and deletion of rectangular solids and cylinders in the manner of many 3D CAD modelers. There is automatic "working" surface detection so objects can be easily created and moved relative to existing objects. Objects drawn "into" other objects create depressions or holes by Boolean subtraction. Boolean union, subtraction and joining are supported. Objects that are created on, or moved into contact with, other objects are automatically linked to that other object in a parent/child relationship so that moving/duplicating takes place in an expected manner without requiring preselection. Optional grids with grid snapping and object snapping are provided for greater precision when necessary. There is unlimited undo. Model and library translation to and from DXF is provided.

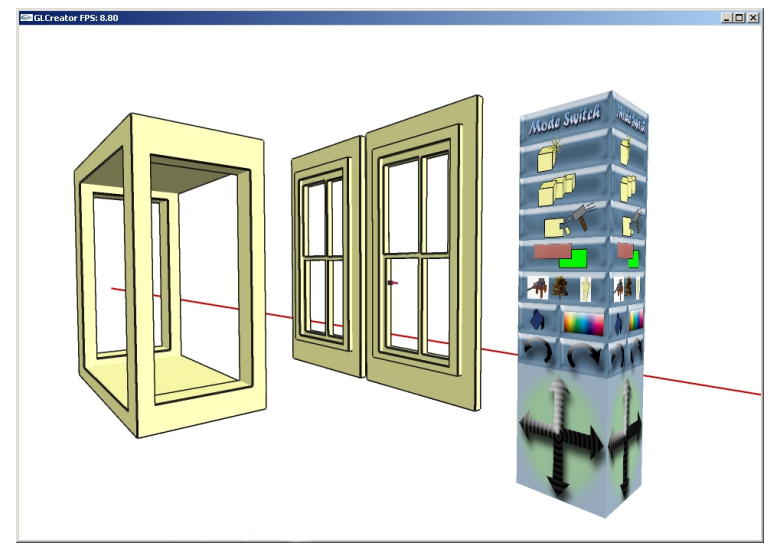

Figure 2: The geometry tools kiosk, and some simple objects created with our modeler.

Use and creation of image mapped library objects is supported. The present library contains objects typical of those contained with many CAD applications. Our intention is to 
extend the use of library objects in preference to creating a more extensive toolbox for geometry creation.

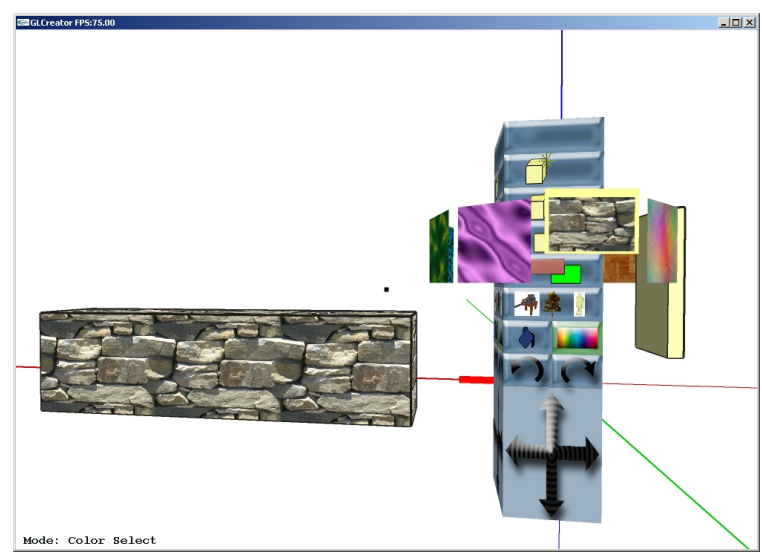

Figure 3: The kiosk with texture selection wheel displayed.

The traditional menu-based toolbox has been replaced with a movable kiosk in order to support natural functionality within a fully immersive paradigm as well as to provide a familiar object within the design space that can help with orientation and sense of scale. The toolbox has an optional "puppy" mode in which it follows the "owner" around, trying to stay in a useful position nearby while not getting in the way. The toolbox also serves as a base for the image/video/library object/environment selection drum discussed later.

Several "skins" are being developed for the kiosk to allow for researching the nature of the kiosk interface.

We are presently implementing optional toolbox voice commands through 3rd party software.

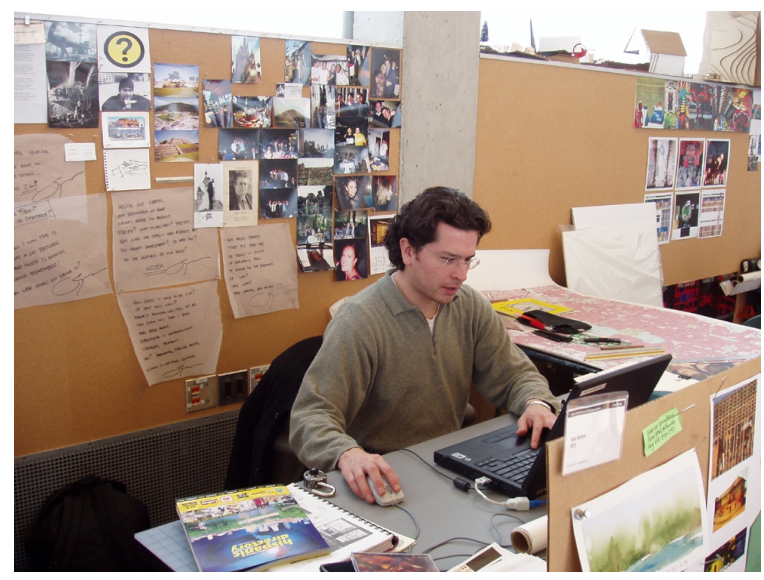

Figure 4: A typical design environment for architecture contains structures such as tables and boards that enable one to surround oneself with images.

\section{Image Environment}

A designer typically requires access to a variety of imagery such as previously drawn sketches, diagrams, notes, tables, site plans, site and other informational photographs and videos, printed specifications, requirements and measurements. Additionally a designer usually keeps sketches of a number of possible design options and a visual record of the design process.

The DesignStation virtual design environment provides a rich environment for images, both still and moving, as well as $3 \mathrm{D}$ geometry. Pictures, textures and videos intended for use within the virtual environment are dropped into a folder. They can then be accessed within the virtual environment by selecting them from a rotating drum on the toolbox kiosk.

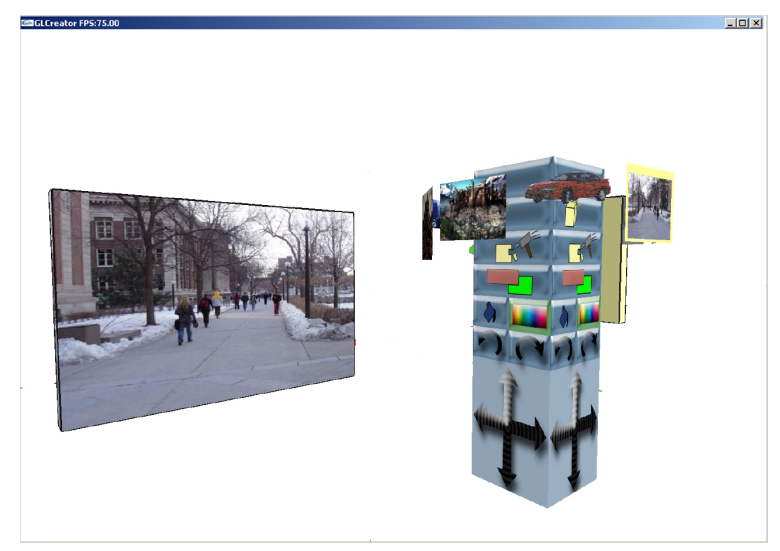

Figure 5: Video sequence selection with the kisok, and an example of a video clip a that might be chosen for inclusion in a design environment.

Images and videos can be placed on any surface, moved, duplicated and scaled. Images and videos can also be placed as standalone objects anywhere within the environment. Transparency and optional billboarding are supported.

Images/videos can serve many purposes within the design environment:

1) to provide detailed information and reference within the DesignStation;

2) to establish a larger contextual environment;

3) to represent real objects, such as people, for scale and indication of activity;

4) as a substitute for geometric objects;

5) for surface representations such as plans and facades;

6) for use as DesignStation "skins"; and

7) for symbolic representation of intention, where the symbolic ideas that can be expressed in a graphic are more appropriate for conceptual design than a geometric object. 


\section{DesignStation/Presentation Area/Critique Space}

The virtual design environment provides for the creation of a designer's work area (DesignStation) that serves to concentrate documents, sketches, plans, videos and other images related to the design and brought into the virtual design environment through the drop folder and kiosk drum previously described. Images and videos can be moved within or between surfaces, rescaled and overlapped within the surfaces of the DesignStation. The materials in the DesignStation can be also be moved/copied onto objects in the design. For example, alternative designs for a facade can be selected and applied from a collection of sketches in the DesignStation.

The work area, minimally composed of one or more short walls or display boards, created by the designer and typically provided through a library object, can be moved to any location, and easily changed to a different configuration. One of our research goals is to determine what types of DesignStation configurations are most useful for various design tasks. We anticipate the development and use of multiple workstation "skins" as well as a variety of complete configurations that support specific different activities of design. The entire state of the virtual design environment, including all aspects of the DesignStation and displayed imagery can be saved and reused.

A further consideration regarding the DesignStation is that the designing space should itself be "habitable". It needs to be capable of providing the physical support for engaging the designer in a sustained effort that includes reflection and analysis as well as creation.

Many of our anticipated DesignStation configurations have both purely virtual components and real components represented alonside each other in the virtual world. Allowing for the representation of real components in the virtual world enables us to incorporate into the environment objects such as stools and chairs that can make inhabiting the virtual space more comfortable and thus permit more sustained attention. Real objects can also provide physical aids, such as a worktable, that a designer can rest arms and hands upon to support fine motor movement such as that required when working on designs at a small scale. A secondary reason for placing real objects within the DesignStation is to improve the quality of the designer's experience of the space by making at least some of the commonly encountered objects in the virtual space physically present.

Of course, during use the designer will want to create objects while moving freely throughout the tracked space as well as while residing at the DesignStation.

In a traditional architecture environment, conceptual work in progress is presented in both process and result, in model and sketches, so that the designer, and others referred to in architectural education as "critics", can evaluate the progress of the design and reflect on options. A second purpose of the DesignStation is to support presentation and critique of a

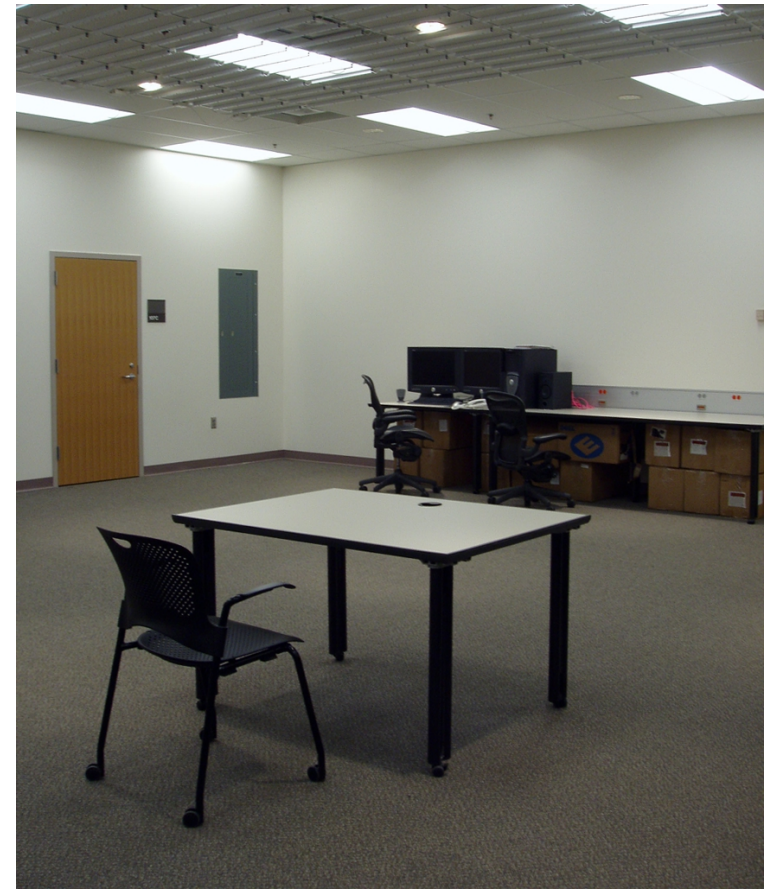

Figure 6: A table and chair in the physical environment.

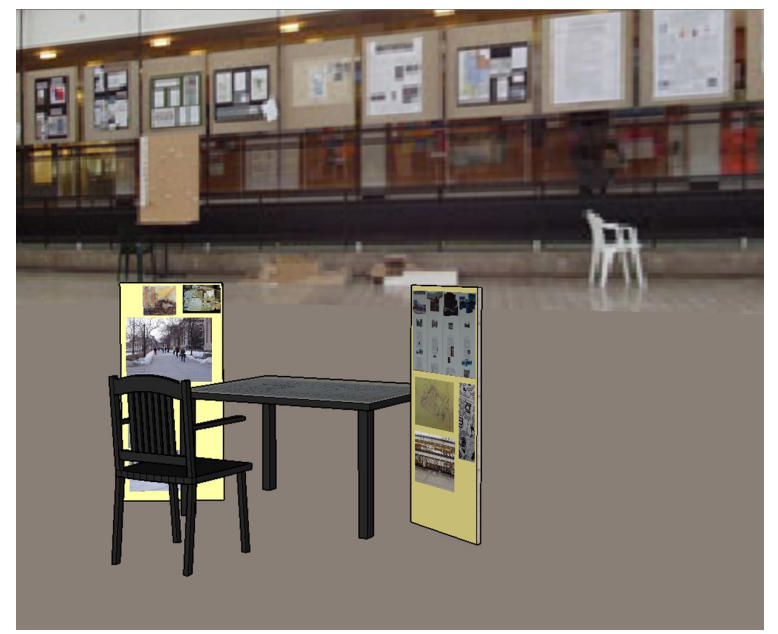

Figure 7: A table and chair in one possible virtual design environment, in this case the interior court of our Architecture building. The courtyard environment is modeled by a large image placed vertically in the background.

design while in process. To support this process the environment provides the ability to take "snapshots" and "videos" of important aspects of a design as seen through the HMD. The images/videos are saved and added to other images on the DesignStation surfaces. 


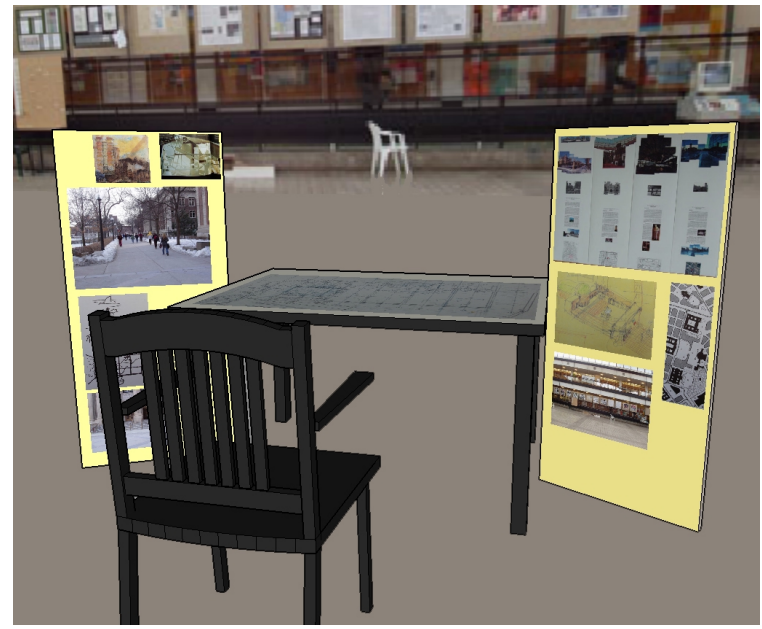

Figure 8: A closeup view of the DesignStation in the virtual design environment, in which images, videos, sketches and other material relevant to the design task are visually accessible on two flanking displayboards.

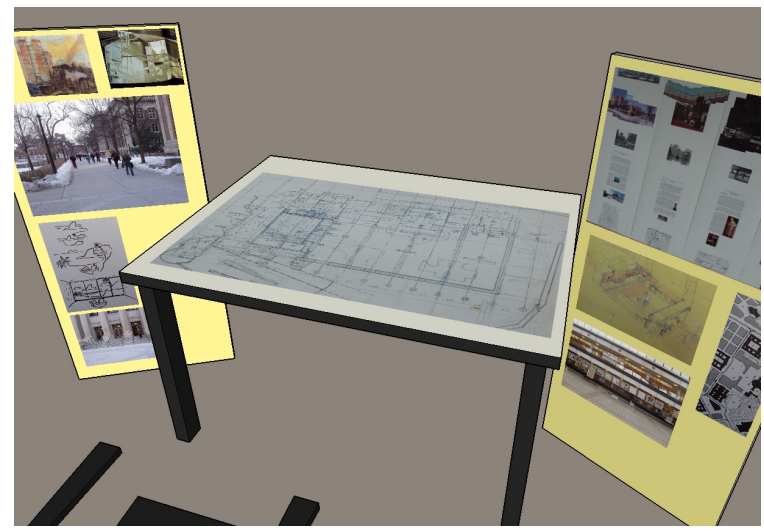

Figure 9: An extreme closeup view of the desktop, upon which a plan view of the contextual environment for the design task has been laid out.

\section{Scaling and Simultaneous Multiple Scales}

While creating and experiencing a design at full scale is an expected goal for an immersive design system, it is not usually the best sole environment for architectural design because it is often necessary to understand and develop the design at multiple scales. Our virtual design environment supports simultaneous multiscale viewing so that conceptual models can be designed and observed at any scale, typically ranging from the scale of an architectural model - a foot or two across - up to actual size.

Simultaneous multiple scales are supported so that a project can be designed, for example, at a relatively small

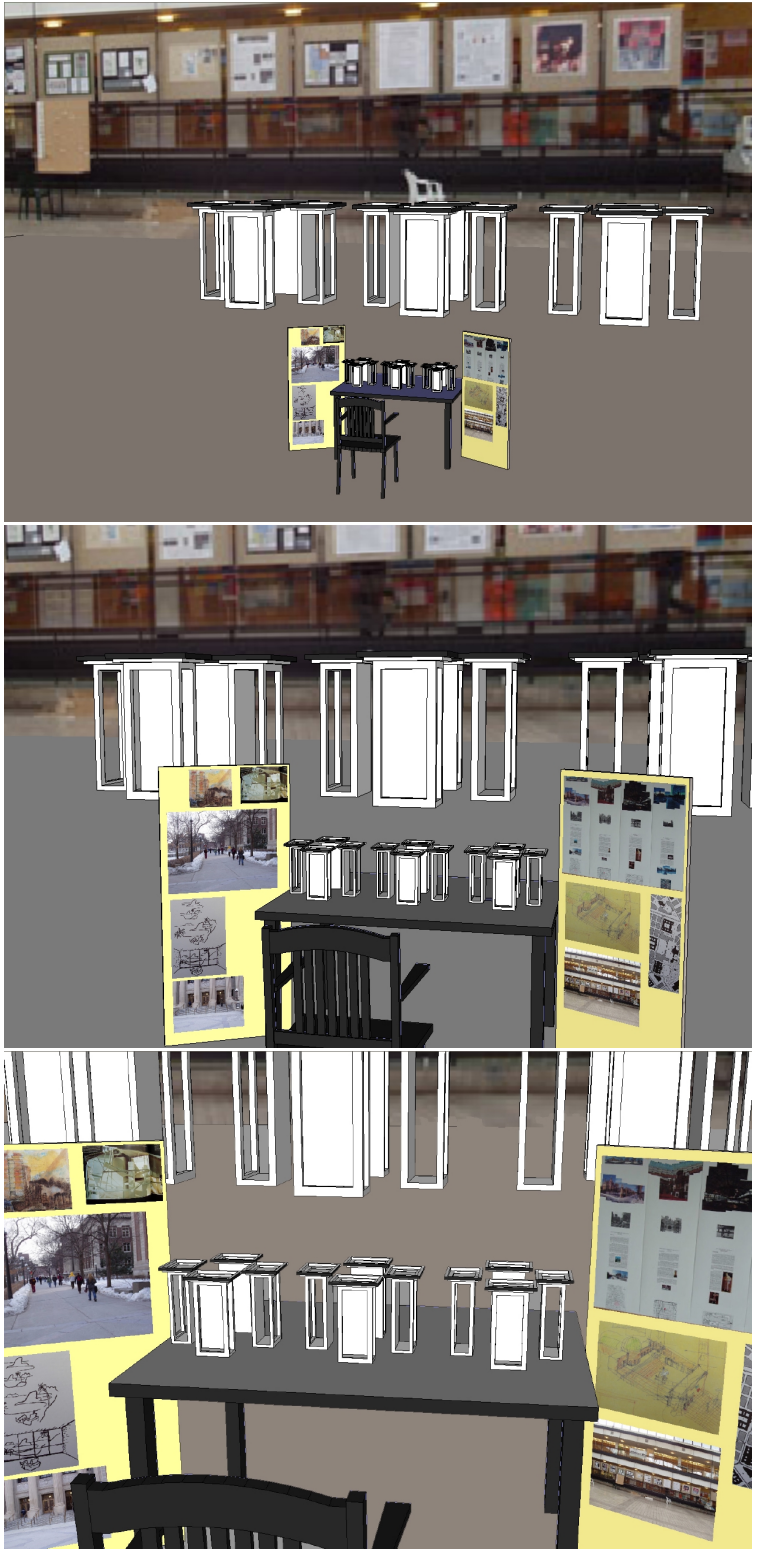

Figure 10: A series of images demonstrating of the use of multiple scales in the context of designing a display system for the interior courtyard of the Architecture building. Interactive design can take place either at the desk or out in space.

scale while simultaneously being inhabited or observed at full scale, or visa versa, with modifications made to the model at one scale appearing simultaneously at the other. For example, one may want to model an entry way at full scale while simultaneously being able to view the design at a scale that allows understanding the relationship of the entry way to the whole house and the surrounding site. In future re- 


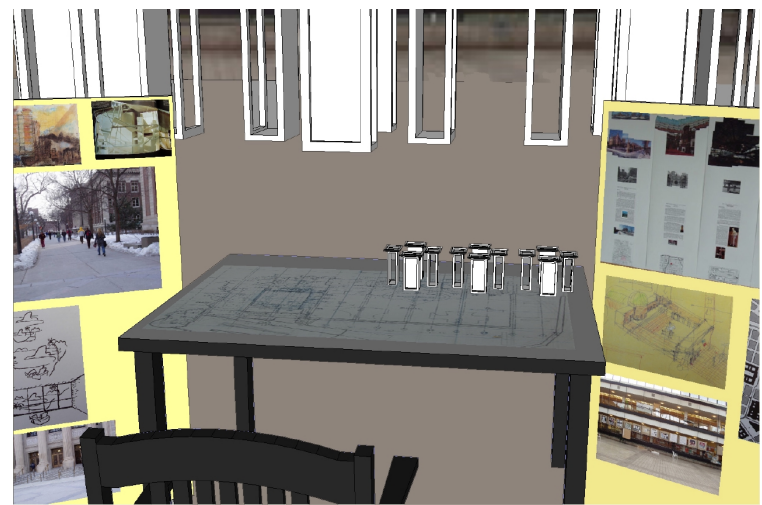

Figure 11: In this image, the model has been scaled to fit in place on the building plan laid out on the desk.

search we intend to explore the effects of body sense while designing at different scales and the perceptual advantages of working at multiple scales simultaneously.

\section{Future Work}

We have several developments presently underway that we expect to incorporate into our system in the near future:

1) We are working on implementing a browser ${ }^{3}$ within the space in order to allow access to standard html documents, either locally or over the web. We would like to enable images/videos/documents/library objects/environments to be drag/dropped directly from the browser into the model. A browser could also permit access to a variety of JAVA applications, such as simple paint programs and analysis tools, that could be useful in supporting a rich design environment.

2) We presently have a standalone "Virtual Graffiti" sketching tool for drawing on surfaces within a virtual environment using tracker output. We intend to make that drawing tool available within the virtual design environment together with the ability to export the resulting images.

3) Images and videos brought into the environment are presently not supported in stereo. Future development will allow for stereo pair images and videos to be placed within the environment. This will include stereo images of the "snapshots" created within the environment and images used to create the overall environment. We anticipate the possibility of creating "virtual holograms", constructed from multiple superimposed images selectively viewed in a position dependent manner.

4) Presently all images and videos come into the virtual environment through the spinning drum provided with the kiosk. We will be adding a "DesignStation" folder for images and videos to be placed directly into the DesignStation, where they can be further arranged by the designer.
Our overall goal is to develop a virtual environment that empowers designers. We intend to refine our system by observing how design is done in the existing virtual design environment and developing new approaches to meet perceived needs. To this end we will be making the system and the space available both to a number of different architectural design studios in the professional architectural graduate program at the University of Minnesota and for use in selected design projects undertaken by local architecture and interior design firms. Design sessions will be videotaped and the design process critiqued together with the designer.

Although our virtual design environment is being developed with the specific needs of architectural conceptual design in mind, we anticipate that this kind of environment could find wider use as well. It could be alternatively thought of as a working environment for the spatial manipulation and creation of images, with the necessary supporting geometric tools. We hope that our future research will involve a wider variety of faculty and researchers who would like to "design" spatially distributed information spaces.

\section{Acknowledgements}

This work was supported by the Graduate School of the University of Minnesota through the McKnight Land-Grant Professorship program, and by a generous gift to the University of Minnesota by Linda and Ted Johnson, through whose efforts the Digital Design Consortium was established. Additional support was provided by a grant from the National Science Foundation, ACI-9875368.

\section{References}

1. L. Anderson. Virtual Graffiti: Three-Dimensional Paint Tools for Conceptual Modeling in Upfront. Mission Method - Madness [ACADIA Conference Proceedings / ISBN 1-880250-01-2], 1992, pp. 127-133.

2. L. Anderson. Film Theory and Architectural Design. Reconnecting [ACADIA Conference Proceedings / ISBN 1-880250-03-9], 1994, pp. 219-227.

3. I.G. Angus and H.A. Sowizral. VRMosaic: Web Access from within a Virtual Environment. IEEE Computer Graphics and Applications, 16(3), May 1996, pp 6-10.

4. D. Bharat. Immersive Modeling Environments. Proceedings of ACADIA 2001.

5. D. Bowman. Conceptual design space-beyond walkthrough to immersive design. In Designing Digital Space (D. Bertol, ed.), Wiley, New York, 1996, pp. 225-236.

6. D. Bowman, L. Hodges and J. Bolter. The Virtual Venue: User-Computer Interaction in Information-Rich Virtual Environments. Presence: Teleoperators and Virtual Environments, 7(5), October 1998, pp 478-493. 
7. A. Bridges and D. Charitos. On Architectural Design in Virtual Environments. Design Studies, 18, 1997, pp. 143-154.

8. J. Butterworth, A. Davidson, S. Hench and T.M. Olano. 3DM: A Three Dimensional Modeler Using a HeadMounted Display. 1992 ACM Symposium on Interactive 3D Graphics, pp. 135-138.

9. D.A. Campbell and M. Wells. A Critique of Virtual Reality in the Architectural Design Process. University of Washington HITL Technical Report R-94-3, 1994. http://www.hitl.washington.edu/publications/r-94-3/.

10. J. Deisinger, R. Blach, G. Wesche, R. Breining and A. Simon. Towards Immersive Modeling - Challenges and Recommendations: A Workshop Analyzing the Needs of Designers. Proceedings of the 6th Eurographics Workshop on Virtual Environments, June 2000.

11. D. Donath and H. Regenbrecht. Using virtual reality aided design techniques for three-dimensional architectural sketching. Design Computation, Collaboration, Reasoning, Pedagogy. ACADIA Conference Proceedings, 1996, pp.201-212.

12. D. Donath, E. Kruijiff and H. Regenbrecht. Spatial Knowledge Implications During Design Review in Virtual Environments ACADIA Conference Proceedings, 1999, pp. 332-333.

13. D. Donath and H. Regenbrecht. Using Immersive Virtual Reality Systems for Spatial Design in Architecture AVOCAAD '99 Conference Proceedings, Brussels, pp. 307-318.

14. A.S. Forsberg, J.J. LaViola Jr. and R.C. Zeleznik. ErgoDesk: A Framework for Two- and ThreeDimensional Interaction at the ActiveDesk. Proceedings of the 2nd International Immersive Projection Technology Workshop, May 1998.

15. L.C. Hill, C. Chiu-Shui and C. Cruz-Neira. Virtual Architectural Design Tool (VADeT). Proceedings of the 3rd International Immersive Projection Technology Workshop, May 1999, pp. 231-241.

16. N. Kukimoto, K. Toizumi, S. Kitsuki, T. Oda, T. Iwasaki. Virtual Environment for Graphic Thinking for Architectural Design. Proceedings of the Virtual Reality Society of Japan, Second Annual Conference, 1999, pp. 323-326.

17. K.H. Lau and M.L. Maher. Architectural Design and Virtual Worlds. Architecture Week, June 7, 2000, pp. T7.1-T7.2.

18. M. Mine. ISAAC: A Meta-CAD System for Virtual Environments. Computer-Aided Design, 29(8), August 1997, pp 547-553.

19. M. Mine, F. Brooks and C. Sequin. Moving Ob- jects in Space: Exploiting Proprioception in VirtualEnvironment Interaction. Proceedings of ACM SIGGRAPH '97, pp. 19-26.

20. H. Regenbrecht, E. Kruijff, D. Donath, H. Seichter and J. Beetz. VRAM - A Virtual Reality Aided Modeller, Promise and Reality. eCAADe Conference Proceedings, Weimar, Germany, 22-24 June 2000, pp. 235-237.

21. M.A. Schnabel, T. Kvan, E. Kruijff and D. Donath. The First Virtual Environment Design Studio. Proceedings of the 19th ECAADE - Education for Computer Aided Architectural Design in Europe Conference, August 2001.

22. M.A. Schnabel Collaborative Studio in a Virtual Environment. International Conference on Computers in Education, Massey, New Zealand, December 2002.

23. B. de Vries, A.J. Jessurun and J.J. van Wijk. Interactive 3D Modeling in the Inception Phase of Architectural Design. Eurographics Short Presentations, 4(7), September 2001, pp.265-271.

24. http://www.sketchup.com

(c) The Eurographics Association 2003. 